\title{
Theoretical Investigation of the Electrocaloric Properties of Lead-free Ferroelectric Ceramic
}

Hend Kacem ( $\sim$ kacem.hend@gmail.com )

Université de Monastir: Universite de Monastir

Ahmad Dhahri

Shaqra University

Mohamed Amara GDAIEM

Universite de Monastir Faculte des Sciences de Monastir

\section{Z. SASSI}

INSA Lyon: Institut National des Sciences Appliquees de Lyon

\section{SEVEYRAT}

INSA Lyon: Institut National des Sciences Appliquees de Lyon

\section{LEBRUN}

Insa lyon

\section{PERRIN}

INSA Lyon: Institut National des Sciences Appliquees de Lyon

\section{J. DHAHRI}

Universite de Monastir Faculte des Sciences de Monastir

\section{Research Article}

Keywords: Lead-free ceramic, Ferroelectric, Theoretical model, Electrocaloric effect, Entropy change, Heat capacity change

Posted Date: December 23rd, 2020

DOI: https://doi.org/10.21203/rs.3.rs-131870/v1

License: (c) (1) This work is licensed under a Creative Commons Attribution 4.0 International License.

Read Full License 


\title{
Theoretical investigation of the electrocaloric properties of lead-free ferroelectric ceramic
}

\author{
Hend KACEM ${ }^{\mathrm{a},{ }^{, *}, \text { Ah. DHAHRI }}{ }^{\mathrm{b}, \mathrm{c}}$, Mohamed Amara GDAIEM ${ }^{\mathrm{a},{ }^{* *},}$, Z. SASSI $^{\mathrm{d}}$, L. SEVEYRAT $^{\mathrm{d}}$, \\ L. LEBRUN ${ }^{\mathrm{d}}$, V. PERRIN ${ }^{\mathrm{d}}$ and J. DHAHRI ${ }^{\mathrm{a}}$
}

\footnotetext{
${ }^{a}$ Université de Monastir, Faculté des Sciences de Monastir, Laboratoire de la Matière Condensée et des Nanosciences, LR11ES40, 5000, Monastir, Tunisie

${ }^{\mathrm{b}}$ Physics Department, Faculty of Science and Humanities in Ad-Dawadmi, Shaqra University, 11911, Saudi Arabia.

${ }^{\mathrm{c}}$ Université de Sfax, Faculté des Sciences de Sfax, Laboratoire de Physique Appliqué, B.P. 1171, 3000, Sfax, Tunisie

${ }^{\mathrm{d}}$ Université de Lyon, INSA-LYON, LGEF, EA682, F-69621, Villeurbanne, France
}

\begin{abstract}
BaTi $_{0.91} \mathrm{Sn}_{0.09} \mathrm{O}_{3}$ (BST) sample was prepared by the solid-state reaction method. The structural, morphological and electrocaloric properties were studied. The sample crystallized in the tetragonal structure with $\mathrm{P} 4 \mathrm{~mm}$ space group. Based on mapping image, the sensitivity and spatial resolution of the different elements in our sample were improved. According to the variation of polarization as function of temperature, our sample had a paraelectric-ferroelectric phase transition, around room temperature. The electrocaloric properties of our sample were investigated using theoretical approaches. The important parameters such as maximum entropy change, relative cooling power and full width at half maximum were explained qualitatively. These results make our sample promising candidate for refrigeration domain.
\end{abstract}

Keywords: Lead-free ceramic, Ferroelectric, Theoretical model, Electrocaloric effect, Entropy change, Heat capacity change.

Corresponding author:*E-mail:kacem.hend@gmail.com Tel: +21692188163

**: E-mail:Gdaiem_mohamed@ hotmail.fr Tel: +21629523640. 


\section{Introduction}

The refrigeration market has grown considerably because of the constant expansion of the industry, rising living standards, and climate change [1]. This has resulted in a lack of control over consumer energy expenditure. It should be noted that the extensive use of refrigeration is a major factor of excessive energy consumption resulting in the depletion of non-renewable energy resources, which exacerbates the effect of global warming. Nowadays, the open debate is mainly focused on the energy transition towards a green development focused on protecting the environment, preserving human health and reducing global warming. The necessity to improve energy performance has become a major concern for industrial and scientific communities. This fragment of innovation has, therefore, become under great pressure to produce more sustainable technological solutions predicated on promising cooling technologies. A few successful techniques are being developed. For example, the thermoelectric technique (Thomson or Peltier, Seebeck effect), solar sorption [1], as well as magnetocaloric (MC) [2,3] and electrocaloric (EC) cooling [4-7]. Compared to MC cooling its main advantage is that the high electric fields necessary for the refrigeration cycle are less costly and much easier to produce compared to the magnetic fields necessary for MC refrigeration [1]. ECE could be defined in adiabatic conditions by the change of temperature when an electric field is applied. In 2006, A. S. Mischenko et al have found a giant ECE in thin films $\mathrm{PbZr}_{0.95} \operatorname{Ti}_{0.05} \mathrm{O}_{3}$ [8]. The disadvantage of this type of material is that they are toxic and require high electric fields, which limits practical applications $[\mathbf{9 , 1 0 ]}$. So far, most inorganic materials with exceptional ECE are lead-based while lead-free ceramics, generally, have a lower ECE [11,12]. Nevertheless, because of worldwide lead limitations, there is a pressing need to create environmentally-friendly lead-free materials. Among the lead-free EC materials are the prototypical $\mathrm{BaTiO}_{3}$, which has a maximum ECE at high temperatures [13-16]. Nevertheless, there is not much research focusing on adjusting their Curie temperature $\left(\mathrm{T}_{\mathrm{C}}\right)$, near room temperature [17-19]. Thus, it is important to continue research to achieve a giant ECE in a leadfree material in ceramic form by applying a relatively small electric field near room temperature. Previous results have demonstrated that there is a significant increase in entropy of the non-polar phase by dint of invariant critical point where multiple phases can coexist.

In this work, we present a detailed study of the structure, morphology and ECE for the BaTi $_{0.91} \mathrm{Sn}_{0.09 \mathrm{O}_{3}}$ sample. Here, ECE was simulated based on a phenomenological model. This simulation allowed us to predict EC properties under different applied electric fields. The theoretical model predicted the experimental results in order to minimize the cost of 
experimental study. Here, different EC parameters were explained such as; example entropy $\left(-\Delta \mathrm{S}^{\mathrm{E}}\right)$, relative cooling power $(\mathrm{RCP})$, heat capacity $\left(\Delta \mathrm{C}_{\mathrm{P}, \mathrm{E}}\right)$ and temperature changes $(\Delta \mathrm{T})$.

\section{Experimental Details}

$\mathrm{BaTi}_{0.91} \mathrm{Sn}_{0.09} \mathrm{O}_{3}$ polycrystalline sample was prepared by the solid-state reaction method. They used high purity precursors with high purity were $\mathrm{BaCO}_{3}, \mathrm{TiO}_{2}, \mathrm{SnO}_{2}$. The initial powder was prepared by grounding starting materials in ethanol with an agate mortar for $2 \mathrm{~h}$. The resulting powder was calcined in two stages: at $900^{\circ} \mathrm{C}$ for $24 \mathrm{~h}$ and then at $1200^{\circ} \mathrm{C}$ for $12 \mathrm{~h}$. Between the two stages the powder was well ground and for the second stage the powder was calcined in pellet form of $12 \mathrm{~mm}$ diameter. The obtained powder was again ground for $2 \mathrm{~h}$ and was pressed under a pressure of 5 tons per $\mathrm{cm}^{2}$ into pellets with a diameter of $8 \mathrm{~mm}$. Subsequently, these pellets were sintered at $1400^{\circ} \mathrm{C}$ for $2 \mathrm{~h}$ to get dense ceramic. The pellet sample was immersed in a thermostatic oil bath. X-ray diffraction (XRD) pattern of the sintered BST ceramic was recorded on a Philips diffractometer using CuK $\alpha$ radiation $(\lambda=1.54056 \AA)$ in the angle range $20^{\circ} \leq 2 \theta \leq 90^{\circ}$. The microstructure was determined, at room temperature, by a scanning electron microscope (SEM) using a TS QUATA 250. The compositions of our sample was obtained by a semi-quantitative analysis performed at $15 \mathrm{kV}$ accelerating voltage using energy dispersive X-ray analysis (EDX). Field emission scanning electron microscopy (FE-SEM) (JSM-7500, Japan) was used to investigate the surface morphologies and element mapping of the studied sample. The hysteresis measurements were determined for different electric fields and different temperatures by current amplifier Keithley 428 and high voltage amplifier TREK Model 20/20C at a switching frequency of $1 \mathrm{~Hz}$. The polarization (P) versus temperature (T) data for different fields was determined from hysteresis loops.

\section{Results and discussion}

To describe the structural properties of our sample, we carried out XRD analysis, at room temperature. Fig.1 shows the dependence of XRD patterns of BST ceramic. It crystallized in the tetragonal structure with P4mm space group with cell parameters: $a=b=6.854$ (2) $\AA$ and $c=17.597(3) \AA ; \alpha=\beta=\gamma=90^{\circ}$. Based on Debye Scherer's formula [20] and Williamson-Hall (W-H) method [21], the mean size of crystallites of BST ceramic was calculated. The values of $D$ obtained using Debye Scherrer's formula and W-H method were 149 and $159 \mathrm{~nm}$, respectively. This difference between the two methods is due to the lattice stress correction term in the calculations. 
Regarding the SEM micrograph, the inset (a') of Fig. 2. (a), shows that our sample featured a relatively dense microstructure. The average particle size of the compounds was estimated using Image $\mathbf{J}$ software. Then, we can adjusted the data obtained with the log-normal function [22]:

$$
f(D)=\frac{1}{\sqrt{2 \pi} \sigma D} \exp \left(-\frac{\ln \left(\frac{D}{D_{0}}\right)^{2}}{2 \sigma^{2}}\right)
$$

where $D_{0}$ and $\sigma$ are the median diameter obtained from the SEM and data dispersions, respectively. The inset (a"') of Fig. 2. (a) shows the dispersion histogram. The mean diameter $<\mathrm{D} \geq D_{0} \exp \left(\frac{\sigma^{2}}{2}\right)$ and stand deviation $\sigma_{D}=<D>\left[\exp \sigma^{2}-1\right]^{\frac{1}{2}}$ were determined using fit results. The average particle size is summarized in Table II. In addition, Fig.3. (b) shows the composition dependence of element mapping of BST ceramic, in order to clearly display the distribution of elements. One can see that all elements are homogenously distributed into the ceramics matrix.

\section{Theoretical considerations}

The present work was undertaken to measure ECE, near room temperature, in BST ceramic. A phenomenological model was applied to predict significant EC properties of our sample.

The variation of $\mathrm{P}$ vs.T and $\mathrm{T}_{\mathrm{C}}$ is presented by [23-25]:

$$
\mathrm{P}=\left(\frac{\mathrm{P}_{\mathrm{i}}-\mathrm{P}_{\mathrm{f}}}{2}\right)\left[\tanh \left(\mathrm{A}\left(\mathrm{T}_{\mathrm{C}}-\mathrm{T}\right)\right)\right]+\mathrm{BT}+\mathrm{C}
$$

Where;

$\mathrm{P}_{\mathrm{i}} / \mathrm{P}_{\mathrm{f}}$ are the initial/final values of Pat ferroelectric (FE)- paraelectric (PE) transition, respectively.

$* A=\frac{2\left[\mathrm{~B}-\left.\frac{\mathrm{dP}}{\mathrm{dT}}\right|_{\mathrm{T}=\mathrm{T}_{\mathrm{C}}}\right]}{\mathrm{P}_{\mathrm{i}}-\mathrm{P}_{\mathrm{f}}}$

$\mathrm{B}$ is $\mathrm{P}$ sensitivity $\frac{\mathrm{dP}}{\mathrm{dT}}$ at $\mathrm{FE}$ state before transition.

$\mathrm{C}=\frac{\mathrm{P}_{\mathrm{i}}+\mathrm{P}_{\mathrm{f}}}{2}-\mathrm{BT}_{\mathrm{c}}$

A result of this phenomenological model, an $-\Delta S^{E}$ caused by variation of the external electric field from $E_{1}$ to $E_{2}$ is given by: 


$$
\Delta \mathrm{S}^{\mathrm{E}}=\left(-\mathrm{A}\left(\frac{\mathrm{P}_{\mathrm{i}}-\mathrm{P}_{\mathrm{f}}}{2}\right) \operatorname{sech}^{2}\left(\mathrm{~A}\left(\mathrm{~T}_{\mathrm{c}}-\mathrm{T}\right)\right)+\mathrm{B}\right) \frac{\Delta \mathrm{E}}{\rho}
$$

where $\rho$ is the mass density of the sample.

At $\mathrm{T}=\mathrm{T}_{\mathrm{C}},-\Delta \mathrm{S}^{\mathrm{E}}$ becomes maximum so that Eq. 3 may be written as follows:

$$
\Delta \mathrm{S}_{\max }^{\mathrm{E}}=\left(-\mathrm{A}\left(\frac{\mathrm{P}_{\mathrm{i}}-\mathrm{P}_{\mathrm{f}}}{2}\right)+\mathrm{B}\right) \frac{\Delta \mathrm{E}}{\rho}
$$

Another very important parameter for magnetic refrigeration is RCP, which present the product of $\Delta \mathrm{S}_{\mathrm{Max}}^{\mathrm{E}}$ and $\delta \mathrm{T}_{\mathrm{FWHM}}$. It is defined as:

$$
R C P=\left(P_{i}-P_{f}-2 \frac{B}{A}\right) \frac{\Delta E}{\rho} \times \cosh ^{-1}\left(\sqrt{\frac{2 A\left(P_{i}-P_{f}\right)}{A\left(P_{i}-P_{f}\right)+2 B}}\right)
$$

Here, $\delta \mathrm{T}_{\mathrm{FWHM}}$ is an expression of the extent of a function, given by the difference between the two extreme values of the independent variable at which the dependent variable is equal to half of its maximum value. It can be defined as:

$$
\delta \mathrm{T}_{\mathrm{FWHM}}=\frac{2}{\mathrm{~A}} \cosh ^{-1}\left(\sqrt{\frac{2 \mathrm{~A}\left(\mathrm{P}_{\mathrm{i}}-\mathrm{P}_{\mathrm{f}}\right)}{\mathrm{A}\left(\mathrm{P}_{\mathrm{i}}-\mathrm{P}_{\mathrm{f}}\right)+2 \mathrm{~B}}}\right)
$$

A polarization-related change of heat capacity is given by:

$$
\Delta \mathrm{C}_{\mathrm{P}, \mathrm{E}}=\mathrm{T} \frac{\delta \Delta \mathrm{S}^{\mathrm{E}}}{\delta \mathrm{T}}
$$

According to this phenomenological model, a change of heat capacity is given by:

$$
\Delta \mathrm{C}_{\mathrm{P}, \mathrm{E}}=-2 \mathrm{TA}\left(\frac{\mathrm{P}_{\mathrm{i}}-\mathrm{P}_{\mathrm{f}}}{2}\right) \operatorname{sech}^{2}\left(\mathrm{~A}\left(\mathrm{~T}_{\mathrm{c}}-\mathrm{T}\right)\right)\left(\tanh \left(\mathrm{A}\left(\mathrm{T}_{\mathrm{C}}-\mathrm{T}\right)\right)\right) \frac{\Delta \mathrm{E}}{\rho}
$$

\section{Simulation}

In order to apply the phenomenological model, the numerical calculations were performed with parameters displayed in Table. 1. Fig. 3 shows $\mathrm{P}$ vs. T for our studied sample under different electric field values, ranging from 0 to $30 \mathrm{kV} \cdot \mathrm{cm}^{-1}$. The symbols signify the experimental data and the red lines indicate the modeled data based on the model given by Eq. 2. We note that the modeled data are in good agreement with the experimental ones. We can point out that $\mathrm{P}$ presents a continuous change, for different electric fields applied, around $\mathrm{T}_{\mathrm{C}}$ and that $\mathrm{T}_{\mathrm{C}}$ increases considerably with the increase of the electric field. One of the main parameters of the ECE is which has been was plotted for different applied electric fields based on Eq. 3. Fig. 4 shows that $-\Delta S^{E}$ is positive for all the applied electric fields and throughout the temperature 
range, which confirms the FE character [26]. $-\Delta \mathrm{S}_{\max }^{\mathrm{E}}$ is an important parameter that is spotted around $\mathrm{T}_{\mathrm{C}}$. These values of for different applied fields are summarized in Table. 2. In the framework of electrocaloric refrigeration, it is essential to take into account two other parameters, having the same importance of $-\Delta \mathrm{S}_{\max }^{\mathrm{E}}$, namely $\mathrm{RCP}$ and $\delta_{\mathrm{TFWHM}}$, which are defined in Eqs. 5 and 6, respectively. All EC parameters are recorded in Table. 2 and are compared to those found in other works [27-30].The $T_{C}$ values for each applied electric field are close to the ambient temperature, which allows us to suggest that our sample can be considered as a promoter candidate for the electrocaloric refrigeration. Fig. 5 shows the variation of the $\Delta \mathrm{C}(\mathrm{P}, \mathrm{E})$ as a function of the temperatures for different electric fields from 0 to $30 \mathrm{kV} . \mathrm{cm}^{-1}$, based on Eq. 8. In this figure, we can see that $\Delta \mathrm{C}(\mathrm{P}, \mathrm{E})$ changes strongly from a negative to a positive value around $\mathrm{T}_{\mathrm{C}}$, which confirms the $\mathrm{FE}$ behavior in our sample [31]. The obtained $\Delta \mathrm{C}_{\mathrm{P}, \mathrm{E}}^{\min }$ and $\Delta \mathrm{C}_{\mathrm{P}, \mathrm{E}}^{\max }$ values of our sample, under different electric fields, are listed in Table. 2 .

To verify the nature of the magnetic phase transition, it is necessary that the plots $\Delta \mathrm{S}_{\mathrm{M}}(\mathrm{T})$, measured indifferent $\mu_{0} \mathrm{H}$, should collapse on a single curve with a second-order phase transition, which suggested by Franco et al. [32,33]. So by analogy with the MCE, the universal phenomenological $\Delta S^{\prime}$ curve can be determined by the normalization of $-\Delta S^{E}[34,35]$ :

$$
\Delta \mathrm{S}^{\prime}=\Delta \mathrm{S}^{\mathrm{E}}(\mathrm{T}, \mathrm{E}) / \Delta \mathrm{S}_{\mathrm{max}}^{\mathrm{E}}
$$

Therefore, to construct the universal curve, it is important to resize the temperature axis, below and above $\mathrm{T}_{\mathrm{C}}$, by a new parameter on two clearly separated reference temperatures, represented by the following equation:

$$
\theta= \begin{cases}\frac{T-T_{C}}{T_{C}-T_{r_{1}}} & T \leq T_{C} \\ \frac{T-T_{C}}{T_{r_{2}}-T_{C}} & T \geq T_{C}\end{cases}
$$

where $\theta, T_{r 1}$ and $T_{r 2}$ are, respectively, the rescaled temperature and the temperatures lower and higher than $\mathrm{T}_{\mathrm{C}}$ of each curve which should satisfy the relation $\Delta \mathrm{S}^{\mathrm{E}}\left(\mathrm{T}_{\mathrm{r}_{1,2}}\right)=-\Delta \mathrm{S}_{\max }^{\mathrm{E}} / 2$.

The curves of $\Delta S^{\prime}(\theta)$ for different applied electric fields are shown in Fig. 6. It is worth noting that all the data are dispersed on a single universal curve, which indicates that the nature of the transition in our sample is second-order [36]. 


\section{Conclusion}

To sum up, $\mathrm{BaTi}_{0.91} \mathrm{Sn}_{0.09} \mathrm{O}_{3}$ sample was prepared by solid-state method. Based on mapping image, the sensitivity and spatial resolution of the different elements in our sample were improved. According to polarization vs. temperature analysis, our sample admits a FE-PE phase transition around room temperature. ECE properties of our studied sample were investigated. The different EC parameters were comparable to those obtained in the literature. This allows our sample to present as a potential candidate in the refrigeration domain. 


\section{Reference}

[1] Matjaz Valant, Progress in Materials Science 57 (2012) 980-1009.

[2] L. Shebanovs, K. Borman, W.N. Lawless, A. Kalvane, Ferroelectrics 273 (2002) 137-142.

[3] M. Tishin, Y. I. Spichkin, The magnetoelectric effect and its applications, Institute of Physics Publishing, Philadelphia, 2003.

[4] S. Mischenko, Q. Zhang, J.F. Scott, R.W. Whatmore, N.D. Mathur, Science 311 (2006) 1270-1271.

[5] H. J. Ye, X.-S. Qian, D.-Y. Jeong, S. Zhang, Y. Zhou, W.-Z. Shao, L. Zhen, and Q. M. Zhang, Appl. Phys. Lett. 105 (2014) 152908.

[6] X. S. Qian, S.-G. Lu, X. Li, H. Gu, L.-C. Chien, and Q. Zhang, Adv. Func. Mater. 23 (2013) 2894.

[7] D. Matsunami and A. Fujita, Appl. Phys. Lett. 106 (2015) 042901

[8] A. S. Mischenko, Q. Zhang, J. F. Scott, R. W. Whatmore, and N. D. Mathur, Science 311, 1270 (2006).

[9] Lu SG, Rozic B, Zhang QM, Kutnjak Z, Pirc R, Lin M, Li X, Gorny L. Appl. Phys. Lett. 97 (2010) 202901.

[10] Correia TM, Young JS, Whatmore RW, Scott JF, Mathur ND, Zhang Q. Appl. Phys. Lett. 95 (2009) 182904.

[11] M. Valant, Prog. Mater. Sci. 57 (2012) 980.

[12] G. Singh, V. S. Tiwari, and P. K. Gupta, Appl. Phys. Lett. 103 (2013) 202903.

[13] Y. Liu, I. C. Infante, X. J. Lou, D. C. Lupascu, and B. Dkhil, Appl. Phys. Lett. 104 (2014) 012907.

[14] G. Singh, V. S. Tiwari, and P. K. Gupta, Appl. Phys. Lett. 103 (2013) 202903

[15] Y. Bai, G. Zheng, and S. Shi, Appl. Phys. Lett. 96 (2010) 192902.

[16] X. Moya, E. Stern-Taulats, S. Crossley, D. Gonzalez-Alonso, S. KarNarayan, A. Planes, L. Manosa, and N. D. Mathur, Adv. Mater. 25 (2013) 1360.

[17] X. Q. Liu, T. T. Chen, M. S. Fu, Y. J. Wu, and X. M. Chen, Ceram. Int. 40 (2014) 11269.

[18] X.-S. Qian, H.-J. Ye, Y.-T. Zhang, H. Gu, X. Li, C. A. Randall, and Q. M. Zhang, Adv. Funct. Mater. 24 (2014) 1300.

[19] Y. Bai, X. Han, K. Ding, and L.-J. Qiao, Appl. Phys. Lett. 103 (2013) 162902.

[20] Dhahri, A., Kacem, H., \& Dhahri, J. (2020). Effect of $\mathrm{La}^{3+}$ substitution on the physical properties of $\mathrm{CaTiO}_{3}-0.15 \mathrm{KNbO}_{3}$-based lead-free ceramics. Applied Physics A, 126(8), 1-10. 
[21] Williamson, G. K., \& Hall, W. H. (1953). X-ray line broadening from filed aluminium and wolfram. Acta metallurgica, 1(1), 22-31.

[22] M.H. Ehsani, T. Raouf, F.S. Razavib, J. Magn. Magn. Mater, 475 (2019) 484-492.

[23] Hamad, M.A, J Adv Ceram 2 (2013) 308-312.

[24] Mahmoud A. Hamad, J. Adv. Dielectr, 3 (2013) 1350029

[25] Mahmoud A. Hamad, AIP Advances, 3 (2013) 032115.

[26] Yang Bai, Guang-Ping Zheng,San-Qiang Sh, Mater. Res. Bull, 46 (2011) 1866-1869.

[27] Mahmoud A. Hamad Appl. Phys. Lett. 102, 142908 (2013)

[28] A Mehmet Sanlialp, Vladimir V. Shvartsman, Matias Acosta, and Doru C. Lupascu, J. Am. Ceram. Soc., 99 (2016) 1-9.

[29] Jinfei Wang, Tongqing Yang, Shengchen Chen, Gang Li, Qingfeng Zhang, Xi Yao, J Alloy Compd 550 (2013) 561-5630

[30] Shijuan Lu, Guorui Chen, Yongcheng Zhang, Zhenmin Zhao, Feng Li, ZonglinLv, Zhenmeng Ma, Dongdong Wang, Chaojing Lu, Shandong Li, Ceram. Int, 44 (2018) 2195021955

[31] Mahmoud A. Hamad, Int J Thermo phys 34 (2013) 1158-1165.

[32] V. Franco, J.S. Blázquez, A. Conde, Appl. Phys. Lett. 89 (2006) 222512.

[33] V. Franco, A. Conde, Int. J. Refrig. 33 (2010) 465473.

[34] M. Bourguiba, M. A. Gdaiem, M. Chafra, E. K. Hlil, H. Belmabrouk, A. Bajahzar, Appl. Phys. A (2019) 125:375.

[35] S. Bouzidi, M. A. Gdaiem, J. Dhahri and E. K. Hlil, RSC Adv., 9 (2019) 65-76.

[36] L.J. Ding, Physica A 471 (2017) 818-824. 
Figures

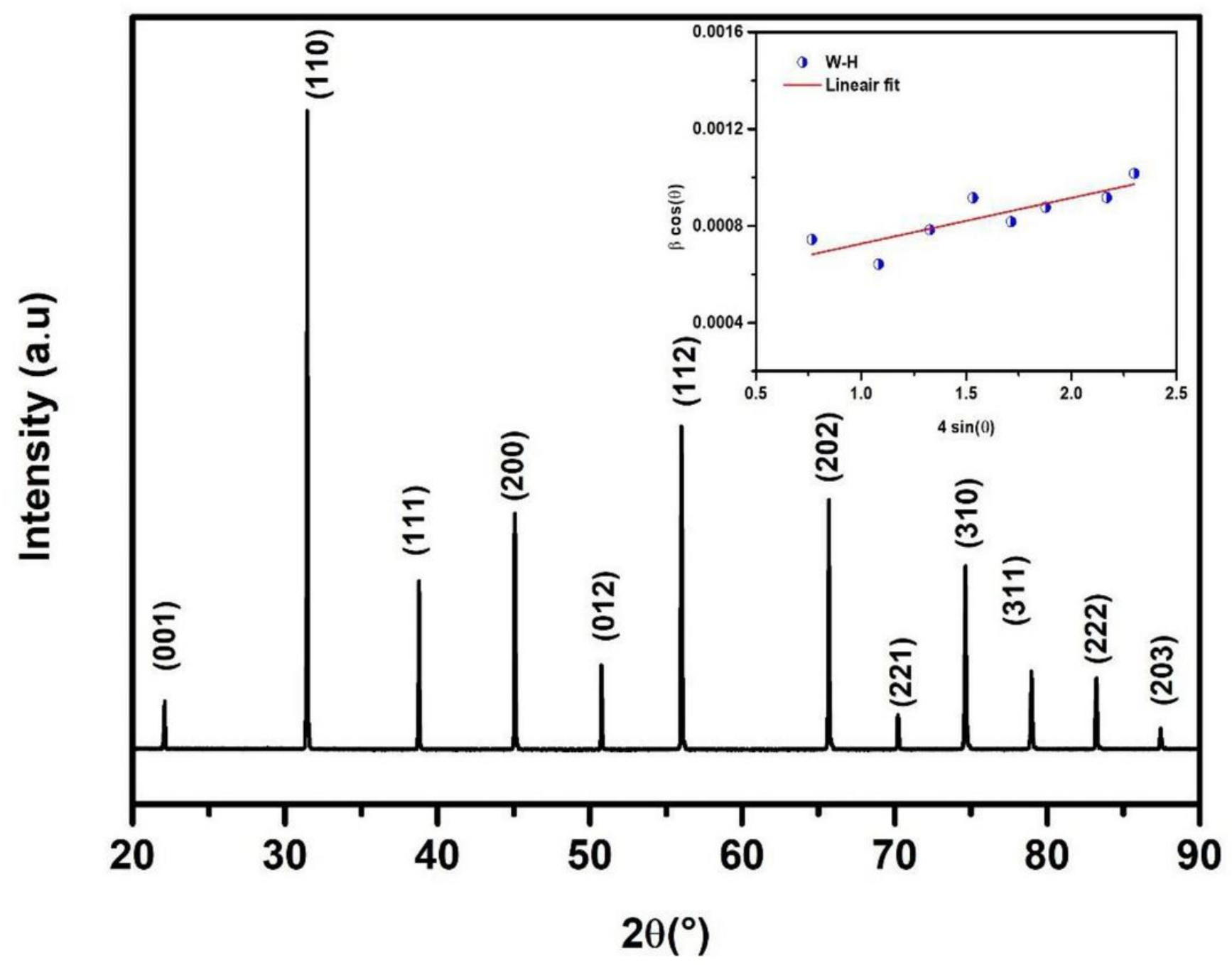

Figure 1

XRD diffraction patterns of BaTi0.91Sn0.0903. The inset shows Williamson-Hall graph of our sample. 

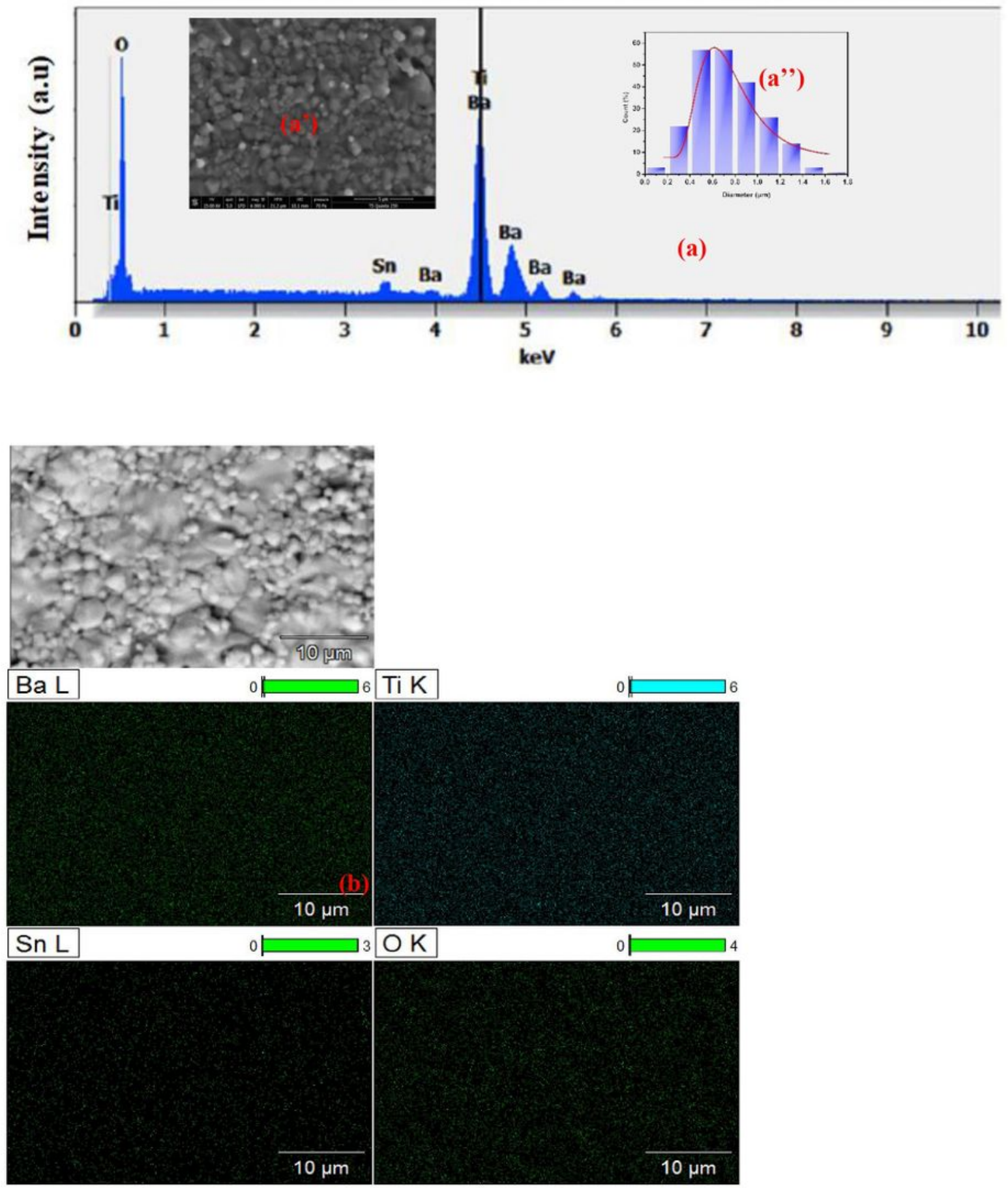

Figure 2

(a) EDX analysis for BaTi0.91Sn0.0903 sample. Insets: (a") of Fig. 2 (a) shows the typical SEM and (a") shows the histogram of the distribution of particles size. Fig. 2 (b) shows EDX maps for Ba, Ti, Sn and 0 elements. 


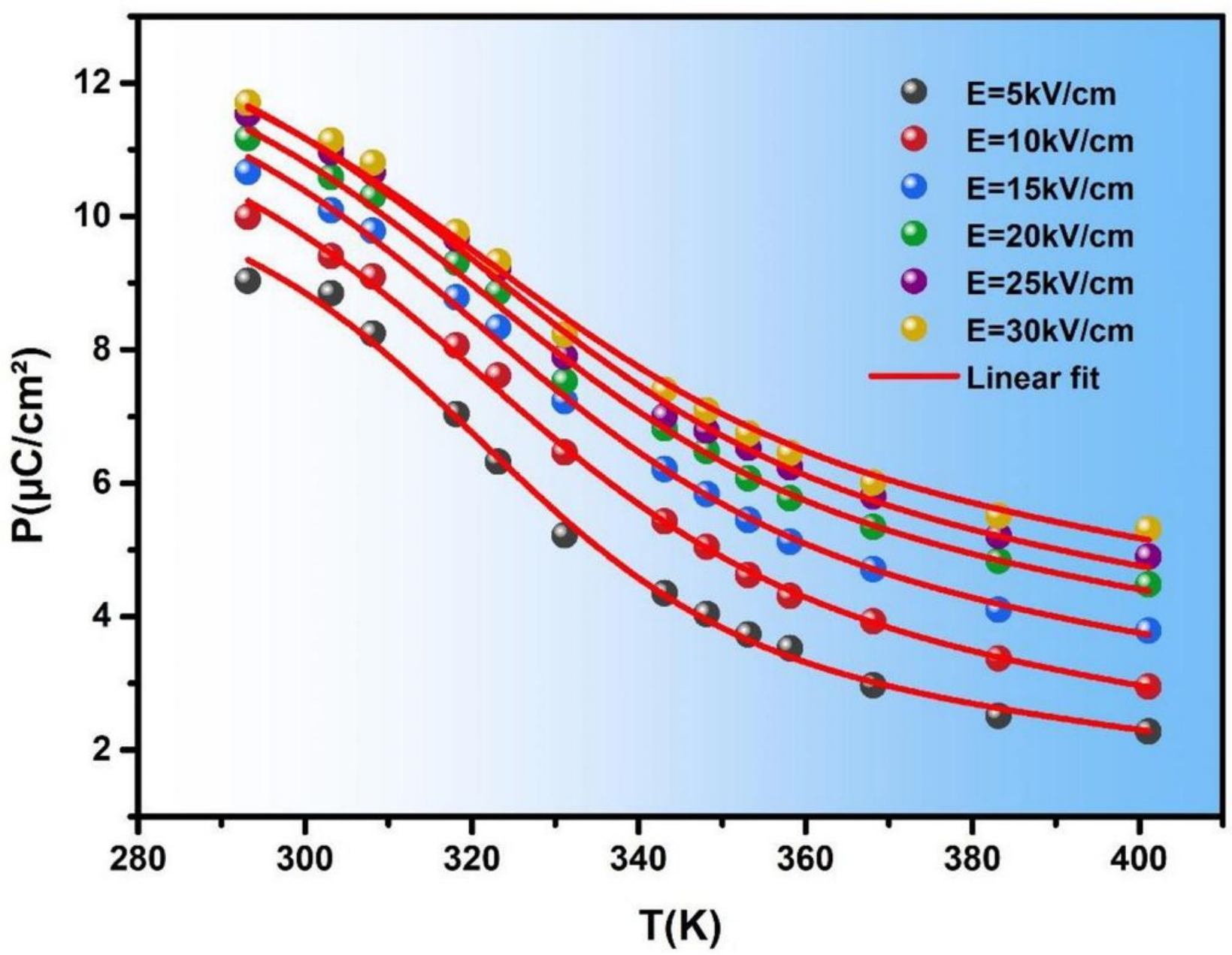

Figure 3

Polarization vs. temperature, under different electric field for BaTi0.91Sn0.0903 sample. The red lines are modeled results by Eq. (3) and symbols represent experimental data. 


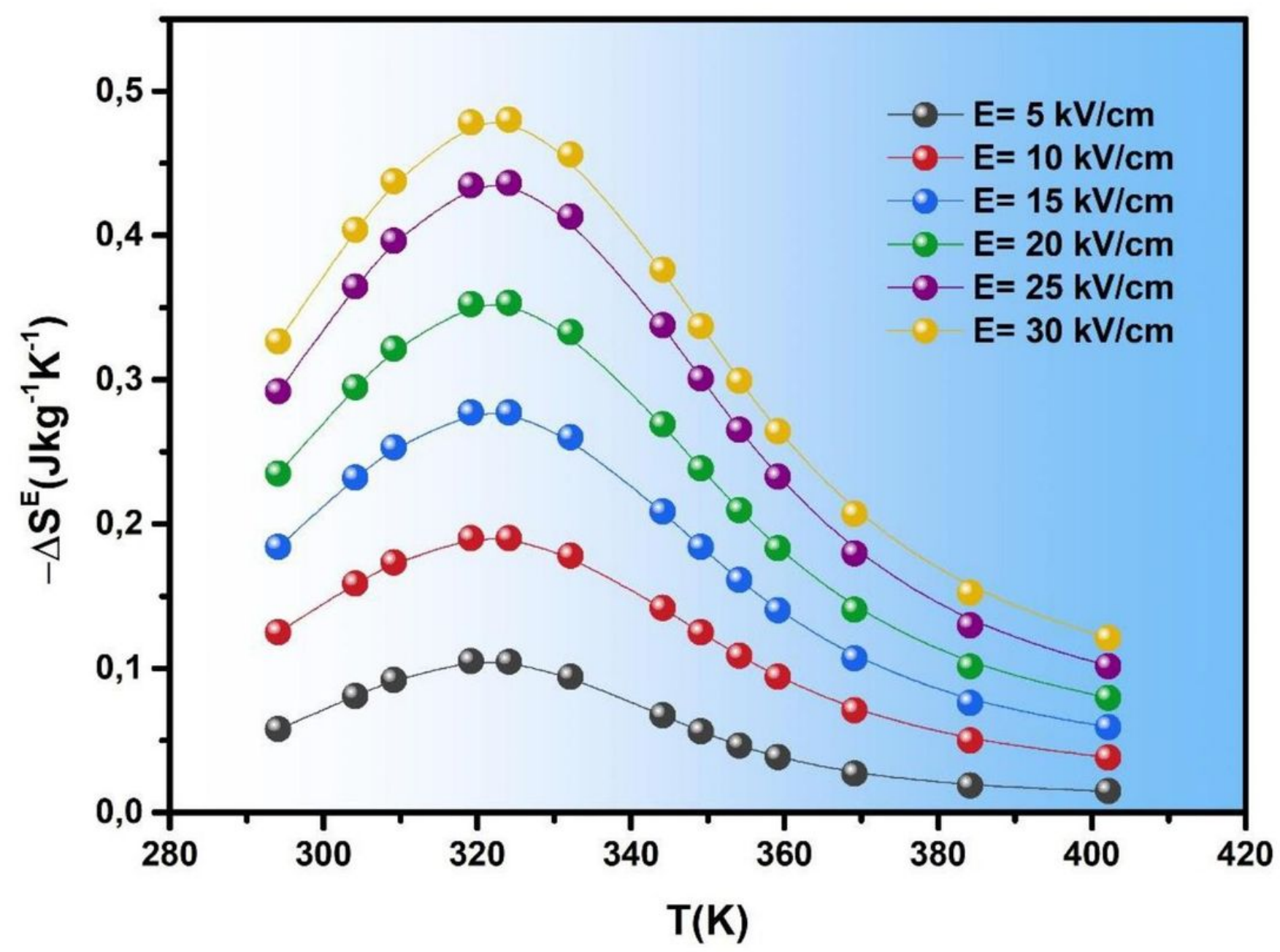

Figure 4

Absolute values of electrocaloric entropy changes vs. temperature, obtained by Eq. (4) at different electric field. 


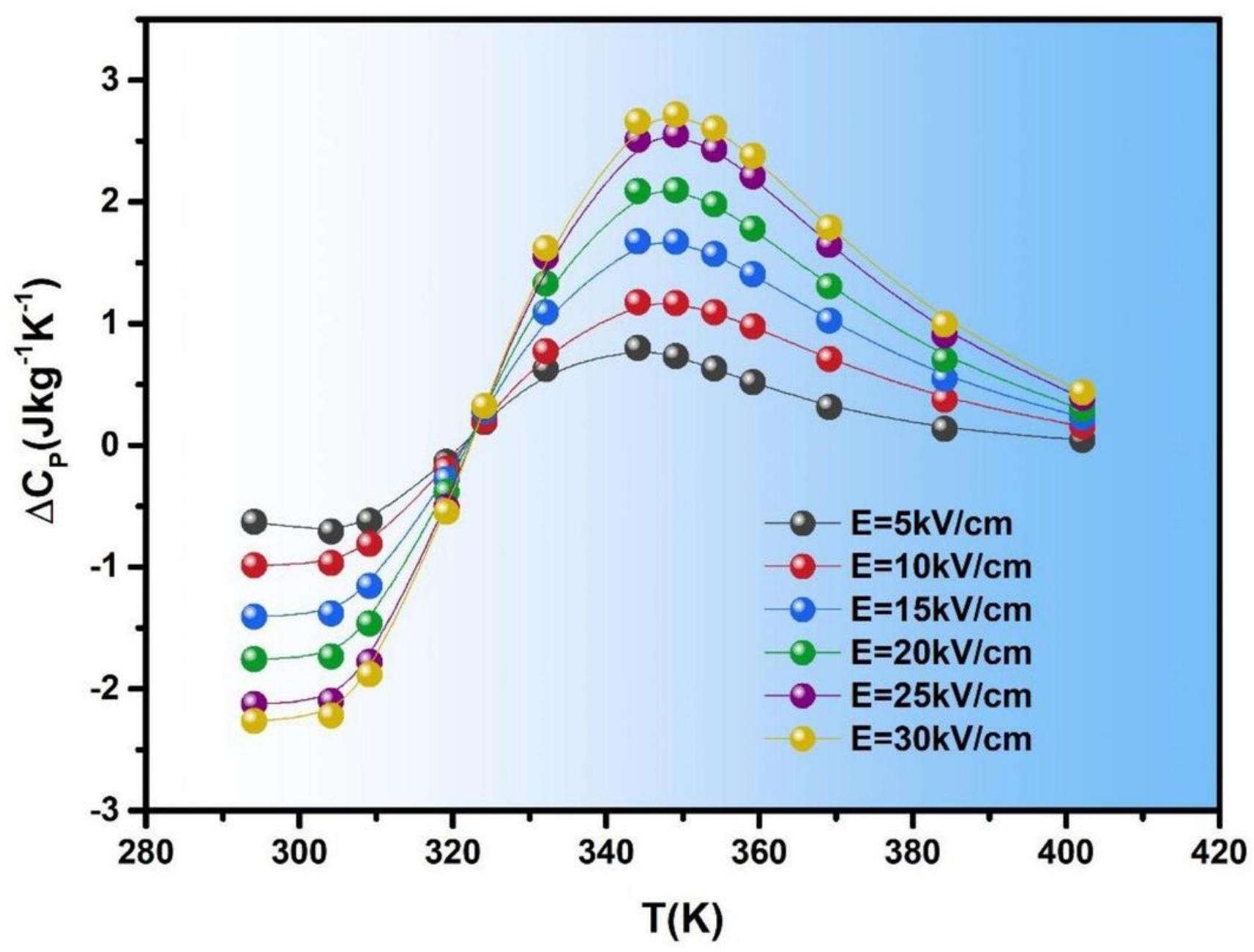

Figure 5

Heat capacity changes versus temperature for BaTi0.91Sn0.0903 sample, obtained by Eq. (8) at different electric field. 


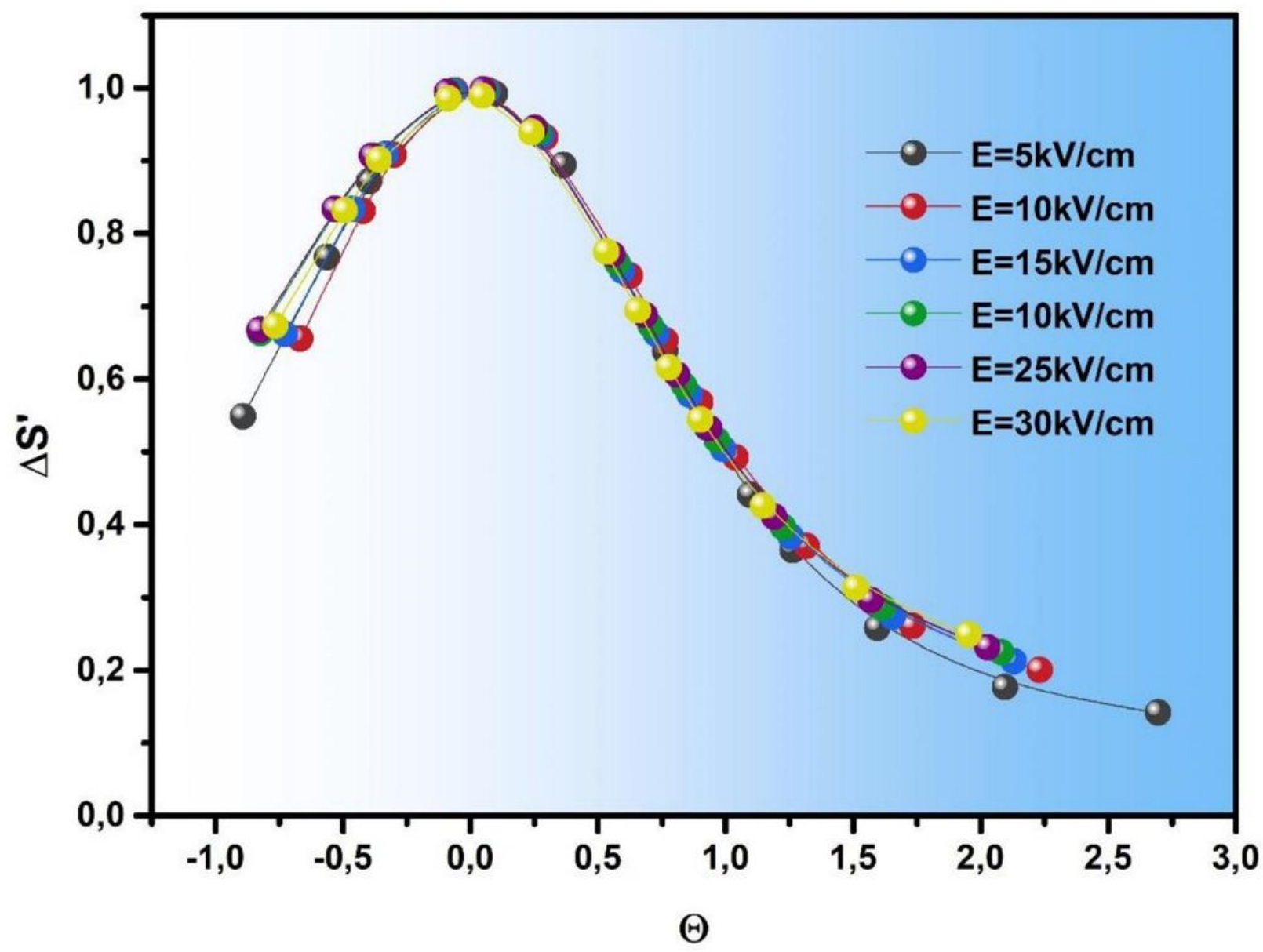

Figure 6

$\Delta S^{\prime}$ vs. $\Theta$ for BaTi0.91Sn0.0903 sample, under different electric field.

\section{Supplementary Files}

This is a list of supplementary files associated with this preprint. Click to download.

- Tables.docx 